Classification

Physics Abstracts

$61.70 \mathrm{~J}-61.70 \mathrm{~L}-62.20 \mathrm{~F}$

\title{
In siru deformation in T.E.M.: recent developments
}

\author{
A. Couret, J. Crestou, S. Farenc, G. Molenat, N. Clement, A. Coujou and D. Caillard
}

CEMES-LOE/CNRS, 29 rue J. Marvig, BP 4347, 31055 Toulouse Cedex, France

(Received February 15, 1993; accepted March 6, 1993)

\begin{abstract}
Résumé. - Au laboratoire, nous étudions les propriétés mécaniques des matériaux par des expériences de déformation in situ réalisées à l'intérieur d'un microscope électronique en transmission. Dans cet article, nous présentons les développements de cette technique mis au point au laboratoire au cours de ces dernières années. De nombreux exemples issus de nos travaux sont cités en illustration. Nous décrivons le procédé de préparation des échantillons et nos porte-objet de déformation. La validité de ces expériences pour expliquer le comportement du matériau massif est ensuite discutée. Une attention plus particulière est portée sur la façon de contrôler la contrainte et sur les éventuels artefacts liés à cette technique. Enfin, nous faisons une revue des potentialités d'analyses qualitatives et quantitatives que possède cette technique.
\end{abstract}

\begin{abstract}
In situ deformation tests in T.E.M. are currently performed in our laboratory on different types of material to study their mechanical properties. Recent developments of this technique are presented in this paper. Many examples from our recent works are demonstrated to support this presentation. The procedure of the specimen preparation and the special straining holders are described in details. The validity of the results to explain the behaviour of the bulk materials is discussed with special attention to the stress control and the possible artefacts. The qualitative and quantitative potentialities of this technique are reviewed.
\end{abstract}

\section{Introduction.}

In 1956, Hirsch, Horne and Whelan [1] were the first to observe in aluminium some movements of dislocations in crystals under thermal stress activated by the beam heating in $100 \mathrm{kV}$ transmission electron microscope (T.E.M.). Two years later, Wilsdorf [2] performed the first in situ tensile experiments in $100 \mathrm{kV}$ T.E.M. at room temperature and observed several dislocation processes proposed previously. These types of experiment did not continue after these pioneer works since at that time it was believed that the observable specimen thickness at $100 \mathrm{kV}$ was too small to give information relevant to the macroscopic behaviour of materials. At the end of the sixties, new in situ deformation experiments on the dislocation behaviour in B.C.C. and F.C.C. metals were undertaken in Japan and France where high voltage electron microscopes were built (for some examples see [3-6]) and in England [7]. Since then, this technique was incessantly used 
and improved. It is worth noting that, as it will be shown in the following, since the beginning of the eighties, most of the in situ deformation experiments have been performed in a low voltage electron microscope.

It is purpose of this paper to take stock of the in situ deformation technique and to focuss attention to recent improvements developed in the Toulouse group. In section 2, some technical aspects will be described. Sections 3 and 4 deal with the stress control and with the validity of the in situ deformation experiments, respectively. Section 5 is an overview of the information obtainable from this technique. Contribution of this technique to the study of the material mechanical properties in relation with other approaches will be discussed in section 6 .

\section{A description of some key technical points.}

\subsection{SPECIMENS.}

2.1.1 Preparation procedure. - To be successful in in situ deformation experiments it is of importance to prepare specimens of highest quality. A satisfactory specimen must be suitable for T.E.M. observations as well as of adequate shape for tensile tests. The perforation must be circular and at the centre of the specimen in order to possess a well-defined stress distribution. The rim of the perforation must be free of cracks; otherwise the deformation could be concentrated at the crack-tips. Since the specimen size (see below) is smaller than that of conventional T.E.M. observations for which the specimen foil is usually a $3 \mathrm{~mm}$ diameter disc, the polished area is smaller and the specimen preparation should therefore be done with extra care. The unpolished parts of the sample need to be protected either mechanically or by wax and this procedure requires some additional caution. Indeed, in the case of a smaller polished area, the circulation of the chemical solution is less efficient and there are some risks that the chemical or electrochemical attack occurs preferentially close to these protections.

The specimen preparation is carried out through two successive stages: the mechanical thinning and the chemical or electrochemical polishing.

i) The mechanical thinning : $400 \mu \mathrm{m}$ thick sheets cut by electric spark erosion are mechanically thinned down to a thickness of $60-70 \mu \mathrm{m}$ by using successive abrasive papers with finer and finer grain sizes. The operation which is at first sight straight-forward has also to be done very carefully since the final success of the preparation depends on the quality of the mechanical thinning. Our experience shows that a thickness of $60-70 \mu \mathrm{m}$ is optimum since it leads after the final polishing to observable areas in T.E.M. which have the good size. Indeed, if the thickness is less than that value, the observable area will be larger and the specimen will be more brittle. Moreover, in this last case, the rim of the perforation is often jagged at the end of the preparation procedure. To have at the end a perforation in the centre of the specimen it is necessary that the two faces are made parallel with a high precision (the accuracy should be $2-3 \mu \mathrm{m}$ ). These different values and conditions should be reproduced in order to have reproductible polishing conditions.

ii) The chemical or electrochemical polishing : Special diaphragm holders adapted to the specimen sizes have to be built. For each type of material, the polishing conditions which are different from those of normal thin foils have to be determined: chemical or electrochemical, chemical solution, temperature of the solution, agitation characteristics and in the case of electropolishing the current intensity and voltage. Some problems often arise from the protections of the unpolished parts and because of that it is sometime necessary to polish the specimen in two steps:

1st step : The unpolished parts are protected and because of these protections the quality of the thin parts may not be good enough. If the quality is good, this polishing is carried on till the formation of a small thin hole, indicating the end of the preparation. If the quality is not good 
enough, this first polishing is stopped when the thin parts are 10-25 $\mu \mathrm{m}$ thick and will be followed by a second step.

2nd step: The protections are removed and the whole specimen is polished in the same conditions as that of the first step till a small hole is formed. This method in two steps is more often used in the case of chemical polishing because the circulation of the solution is less efficient.

2.1.2 Shape. - Depending on the test temperature and on the mechanical strenght, different types of specimen are used. In the low temperature-low strength range, rectangular specimens (Fig. 1a) are glued on copper grips which can be fitted in the specimen holders. In the high temperature-high strength range, because the glue is not strong enough and because the glue evaporation can contaminate the specimen surface, mechanically anchored specimens (Fig. 1b) have to be prepared. Because the procedure to prepare this second type of specimen is obviously more material and time consuming and because the handling of these specimens is more critical, specimens of the second type are used only when absolutely necessary. The maximum temperature at which specimens of first type can be used depends on the material strength and ranges between $200{ }^{\circ} \mathrm{C}$ and $500{ }^{\circ} \mathrm{C}$.
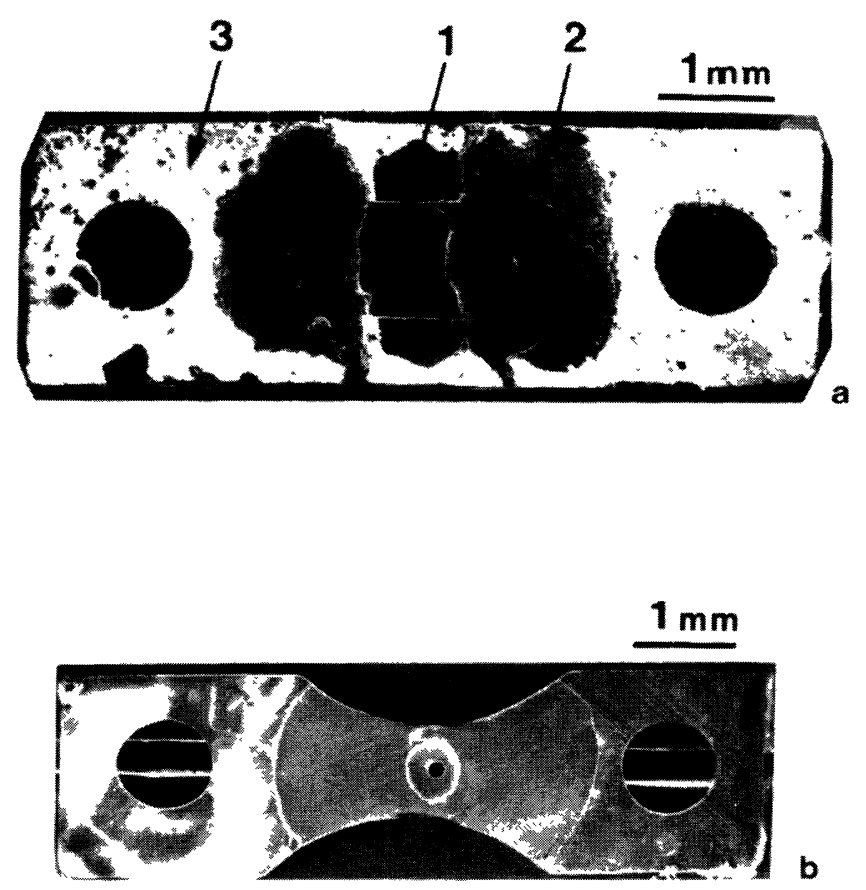

Fig. 1. - Views of specimens, a) low temperature-low strength rectangular specimens 1) specimen, 2) glue, 3) copper grip, b) high temperature-high strength mechanically anchored specimens.

2.1.3 Orientation. - When experiments are performed on single crystals, the specimen orientation in chosen according to the physical problem under investigation. First, the tensile axis is determined to favour the studied glide system by applying the Schmid's law. After that, the choice of the foil plane is the result from a compromise between the following considerations. Different 
diffraction conditions should be reached easily to observe the defects and if possible to characterize them. The slip plane must be inclined with respect to the observation plane in order to image the movement of defects. The line direction of the dislocation segment which are studied should not be too parallel to the foil plane otherwise there is a risk that dislocations take some artefact directions as a result of their shortening line length driven by the lowering of their self energies.

2.2 EQUIPMENT. - To perform in situ deformation experiments it is necessary to use a special equipment adapted to the T.E.M..

2.2.1 Straining holders. - The first step towards being able to build straining holders is to equip a T.E.M. with an objective lens with a sufficient large gap between the pole-pieces. The gap needs to be at least $10 \mathrm{~mm}$ in order to allow for the required tilting of the specimen holder.

i) A cooling straining holder adapted to the JEOL 200CX : Figure 2 shows a general view (a) and an enlargement (b) of the tip of the cooling straining holder. The cooling is achieved via the circulation of gaseous nitrogen in the fixed jaw (8). The nitrogen tank (1) is also used as a vacuum chamber to isolate the cryogenic fluid. (2) is the nitrogen inlet. The pressure in the tank and the gas flow are regulated by a safety valve (3) and by an adjustable leak adapted on the exhaust (4), respectively. The temperature is measured by a thermocouple in the fixed jaw. (5) is the connection for the thermocouple. The accuracy of the temperature measurements by the thermocouple has been checked by observing a phase transition in a $\mathrm{CuZnAl}$ shape memory alloy. This holder works between $100 \mathrm{~K}$ and the room temperature with an accuracy on the temperature regulation of $\pm 10 \mathrm{~K}$ and an autonomy of $45 \mathrm{mn}$ between two fillings. To improve performance (minimum temperature, regulation and autonomy), a straining device cooled down with gaseous helium is now being built in our laboratory.
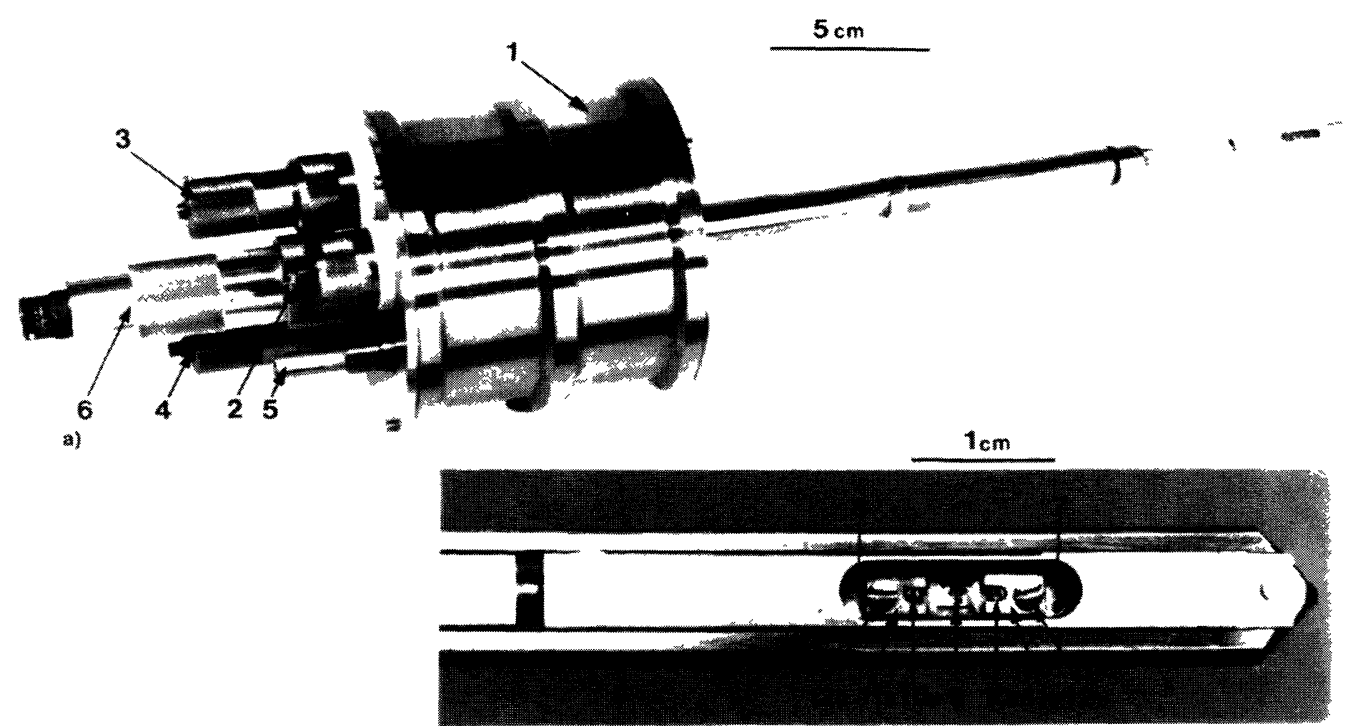

b)

Fig. 2. - Views of the cooling straining holder a) general view, b) enlargement of the tip. 
The deformation system is an inversed one. The deformation is applied by a micromotor (6) which pushes on the mobile jaw (7) (characteristics of the micromotor : $7800 \mathrm{t} / \mathrm{mn}, 12 \mathrm{~V}, 20 \mathrm{~mA}$, reduction ratio : 1/2050). The mobile and fixed jaws are in thermal contact. The specimen (9) is moored on two specimen anchorages $(10, \mathrm{a} \& \mathrm{~b})$ and fixed by some specimen securing plates $(11, a \& b)$ and screws $(12, a \& b)$. During cooling, a spring keeps the two jaws in contact and thus protects the specimen from a deformation resulting from the temperature drift.

ii) An heating straining holder adapted to the JEOL $200 \mathrm{CX}$ : This holder which is inspired from that presented in [8] has already been partly described in [9]. Figure 3 shows a general view (a) and an enlargement (b) of the tip of the heating straining holder. The heating by radiation is obtained by applying a power to a resistor wire (5) which is wound in an alumina multibore tube (6). When it is in place, the specimen (7) is enclosed in a tantalum double screen which is visible in these pictures. The deformation is applied by a micromotor reductor (1) identical to that of the cooling holder which is pulling on the mobile jaw (3). The specimen is anchored between the mobile and fixed (4) jaws. A clutch (2) allows the mobile jaw and therefore the specimen to be free during the heating and to avoid a deformation resulting from the temperature drift. This holder works between the room temperature and $1000^{\circ} \mathrm{C}$. A heating holder with a tip similar to that one adapted to the $3 \mathrm{MV}$ electron microscope of the laboratory has been recently built.
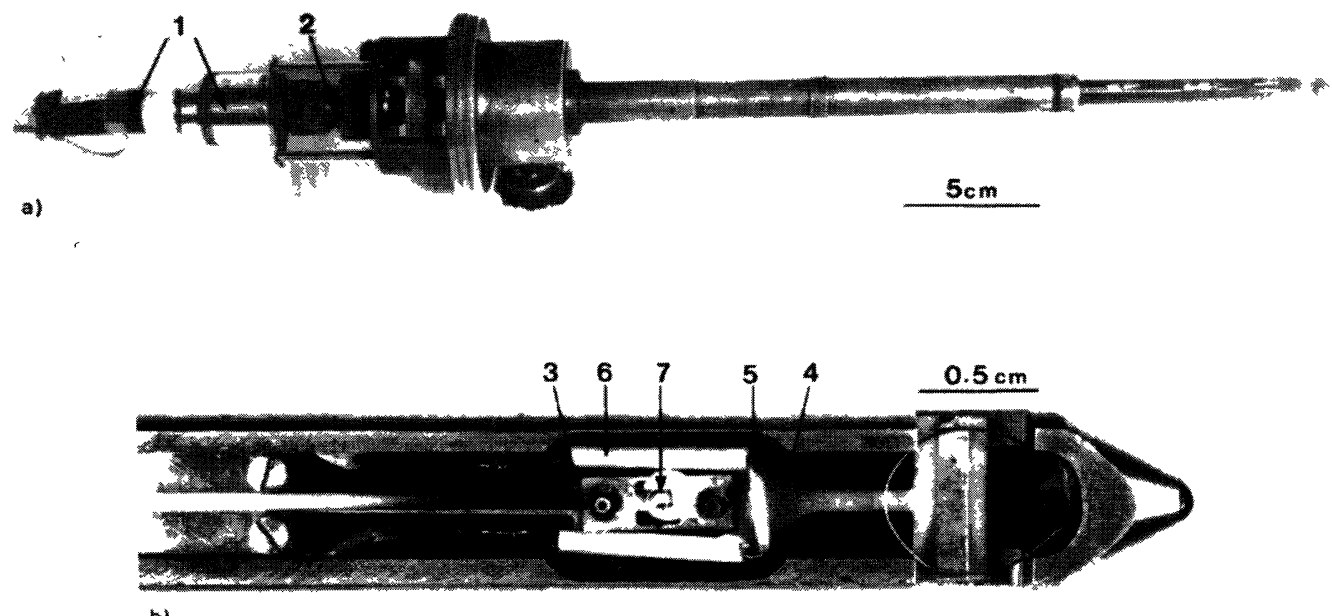

b)

Fig. 3. - Views of the heating straining holder a) general view, b) enlargement of the tip.

The temperature is not measured by a thermocouple because it has been observed that this method is not very accurate in the case of heating by radiation. A curve giving the temperature of the thin parts as a function of the current intensity applied to the resistor has been established by melting some metals and alloys and by observing some phase transitions. With this curve it is therefore possible to apply to the resistor the current corresponding to the required deformation temperature. This method has been found to be very reproductible.

iii) A remark about double-tilt straining holders : Since the deformation of materials with a high sensitivity of the stress to the strain rate (intermetallics for instance) is often unstable under a 
constant stress, the best results have been obtained by using a very hard straining holder, which allows us to deform microsamples at constant strain rate and to observe the relaxation effects at constant total elongation. This requires a very strong straining rod which cannot be coupled with a second tilting device. A double tilt holder is useful in studying bicrystals since it makes possible to image both crystals at the same time [10]. A second tilt is however not necessary in single phased materials and in most coherent two-phased materials with strong epitaxy relations.

2.2.2 Video set. - The dynamic sequences are recorded on a video system. The best solution is to put a transparent fluorescent screen under the camera chamber and to film this screen with a low dose video camera coupled with an amplifier. The video signal is controlled by a TV monitor and sent to a 3/4 inch U-matic magnetoscope. The video camera must record an area approximately equal to half the size of the photographic plate of the microscope because with this value a good resolution can be obtained with both systems at the same magnification and the control of the observed area is made easy when it is switched between the TV monitor and the screen.

The video films are further analysed frame by frame and it is now possible to enhance the quality of the pictures using an image processor.

2.3 DESCRIPTION OF AN EXPERIMENT. - When everything is in place after a first brief checking of the specimen quality, the specimen will be brought at the deformation temperature.

After that, the mobile jaw is moved till the system is under load. Some movements of the bend contours indicate that the specimen is under load and that the specimen is ready to be plastically deformed. When some movements of defects are observed, the micromotor is stopped and the deformation is recorded. That means that our in situ observations are in fact always resulting from relaxation and the stress level corresponds to the elastic limit of the deformation system under investigation.

If necessary, after the in situ experiment, specimens can be observed post-mortem with a double tilt holder in order to further characterize some aspects of the deformation.

\section{Control of the stress.}

3.1 CONTROL OF THE LOCAL STRESS AXIS. - Using a finite element approach, the stress field in the microsample during an in situ deformation experiment has been calculated recently in [11]. The position of the local major principal stress axis within the entire critical zone surrounding the polished hole has been determined for two cases which are representative of two possible polished hole configurations. Figure 4 shows the corresponding results for the case in which the hole is in the centre of the microsample. The higher stress levels have been found in the area where the hole rim is parallel to the tensile direction and the occurence of different slip systems can be explained by applying the Schmid's law. The stress tensor applied to the specimen is well reproduced in this area. For those areas where the edge of the perforation is not parallel to the global tensile axis, the local stress distribution can be worked out from such calculations.

These results are consistent with what is generally occuring during a test. For instance, to study the non-compact glide of aluminium [9], the specimen orientation was chosen in such a way that the glide in an octahedral compact plane was not activated. In the area where the hole edge is parallel to the tensile axis direction, this glide was locally inactive whereas it was activated in the upper and lower parts of the specimen.

3.2 LOCAL STRESS MEASUREMENTS. - The distribution of stress in an in situ tensile sample is inhomogeneous and the local stress is not directly given by the external stress applied to the 


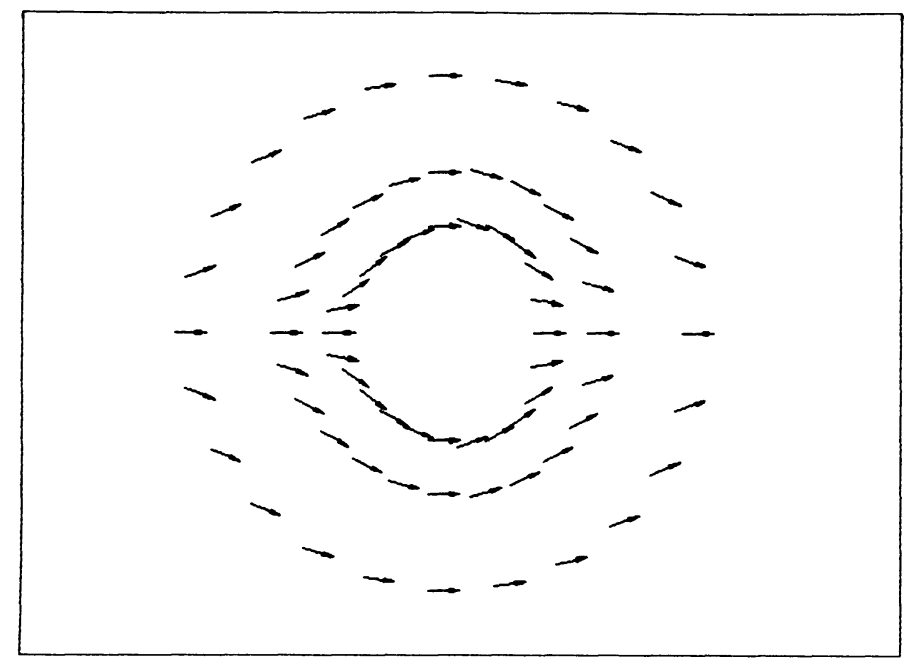

Fig. 4. - Orientation of the major principal axis in a microsample for a specimen in which the hole is in the centre.

sample. It is therefore necessary to evaluate the local stress acting on mobile dislocations. This can be done by measuring the radius of curvature of dislocation segments which are anchored by some obstacles. When a dislocation anchored at both extremities and submitted to a shear stress is in equilibrium, the configuration is described by the following relation:

$$
\begin{aligned}
& \sigma=\frac{\tau}{b R} \quad \text { where : } \quad \sigma \text { is the local stress, } \\
& \tau \text { is the line tension, } \\
& b \text { is the Burgers vector, } \\
& R \text { is the radius of curvature. }
\end{aligned}
$$

It is however possible to apply this formula to the case of a moving dislocation which is not exactly in equilibrium, provided the life time of the anchoring points is long enough. This point has been discussed in [12] in the limit and the complex case of the cube glide in the $\gamma^{\prime}$ phase of a superalloy.

Some evaluations of the line tension can be found in the literature: see for instance [13] for a treatment within isotropic elasticity. For the anisotropic case, the line tension can be obtained as a function of the dislocation character from calculations (see for instance the calculations of Douin in the cases of the octahedral and cube planes of $\mathrm{Ni}_{3} \mathrm{Al}$ in [14] and [15], respectively).

During the deformation, some dislocations having a given character are submitted to a stronger frictional force and thus their glide is slower than the others; in such cases a different method can be used which is based on the same general principle as the previous treatment [16]. In this case, the rapid movement of the mobile parts of the dislocation loop leads to the formation of dipoles composed of the relative immobile segments. The stress is deduced from the measured critical width of the dipole which is assumed to be equal to twice the value of the radius of curvature. This method is interesting since the measurement accuracy of the dipole width is better than that of the radius of curvature. 
3.2.1 Measurements. - The true values of radii of curvature and critical dipole widths are deduced from the measured, projected ones by using some geometric correction factors. In the most general case, it is possible to use the relation given in [17]:

$$
R_{\mathrm{t}}=R_{\mathrm{m}} \frac{1}{\cos ^{2} \alpha}\left(\cos ^{2} \omega+\cos ^{2} \alpha \sin ^{2} \omega\right)^{3 / 2}
$$

where: $\quad R_{\mathrm{t}}$ is the true radius in the slip plane,

$R_{\mathrm{m}}$ is the radius measured on the screen,

$\alpha$ is the angle between the slip and observation planes,

$\omega$ is the angle between the direction of the measurement of

the radius and the intersection of the slip and observation planes.

As it will be discussed in section 4 , the distance between the free surfaces and curved dislocations or dipoles should be larger than $R$. If possible, to obtain a reliable value of the local stress, it would be better to average at least 10 radius measurements on moving dislocations during the same short video sequence. In the case of small values of $R(\leq 50 \mathrm{~nm})$, it is necessary to image curved dislocations either in bright field under $+g$ and $-g$ diffraction conditions, or in weak-beam conditions (see for instance [12]).

Usually, the local stress measurements are not aimed to measure the yield stress but to check that in the thin foil the bulk behaviour is well reproduced. Therefore, even if the accuracy of the absolute value is sometimes not very good (owing to the uncertainties of $R$ and of the line tension expression), the relative variation of the local stress with temperature is often significant enough to check this point. Local stress measurements have been successfully performed in several types of material. In the case of the prismatic glide of beryllium, the yield stress anomaly has been reproduced by this technique [18-20]. In the case of the prismatic glide of titanium, as shown in figure 5, both the CRSS macroscopic variations versus temperature for two types of titanium with different impurity contents and the hardening effect of impurities have also been reproduced in the thin foil [21, 22]. The method of the critical width of the dipole has been applied in several cases (see for instance: [16, 23, 24]). In the anisotropic case of the cube glide of the $\gamma^{\prime}$ phase of a superalloy, local stress measurements performed between $120 \mathrm{~K}$ and $1050 \mathrm{~K}$ have indicated a yield stress anomaly which has been further confirmed by a careful analysis of the corresponding literature $[12,25]$.

\section{Validity of in situ deformation experiments.}

The problem of the relevance of the microscopic observations to explaining the behaviour of the bulk material is often raised. It is the aim of this part to discuss this point only for the case of in situ deformation experiments. This problem has been already discussed in [26] for the case of high voltage electron microscopy and more recently in [27, 28]. During an in situ experiment, the thickness of the observed area varies between $100-500 \mathrm{~nm}$ at $200 \mathrm{KV}$ and between $500-1000 \mathrm{~nm}$ at $1 \mathrm{MV}$. For comparison, in the case of high resolution pictures the thickness is about $5 \mathrm{~nm}$ whereas it is about 10-200 $\mathrm{nm}$ in case of post-mortem observations under weak-beam conditions. In a first section, a non-exhaustive list of possible artefacts is drawn up and in a second section, different ways to check the validity of the observations are described.

\section{1 POSSIBLE ARTEFACTS.}

4.1.1 Image forces. - Dislocations in thin foils are submitted to image forces which result from boundary conditions (zero stress field) at the surfaces. Image forces have been evaluated for different characters of dislocations under different orientations (for a review see [26]). 


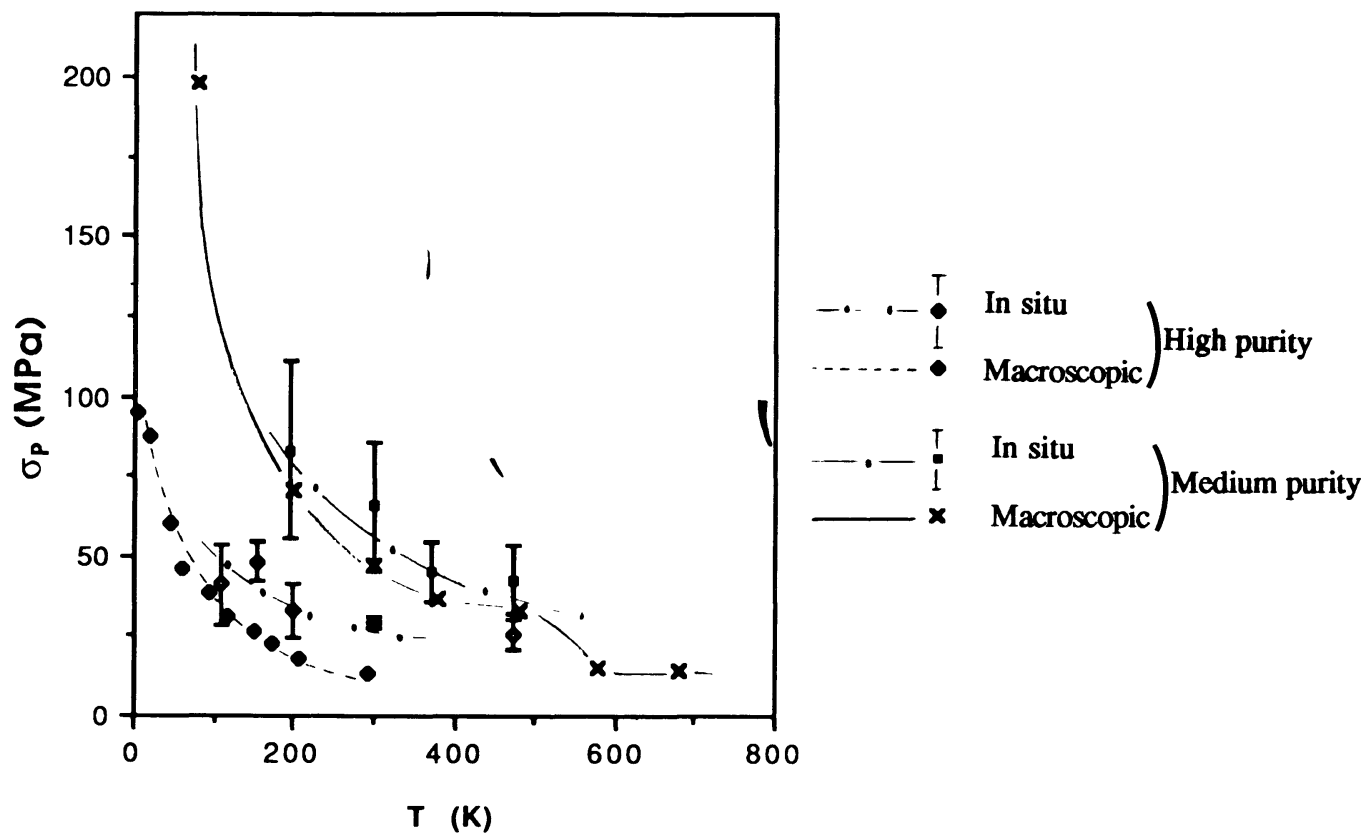

Fig. 5. - Variations of the local stress measured in situ and of the macroscopic CRSS versus temperature for two types of titanium with different impurity contents.

An order of magnitude has been calculated in the case of a screw dislocation lying parallel to the foil plane [28]. In this case, the image forces are given by:

$$
\begin{aligned}
\sigma_{\mathrm{i}}=\frac{\mu b}{4 \pi d} \quad \text { where : } \quad & \sigma_{\mathrm{i}} \text { is the image force, } \\
& \mu \text { is the elastic modulus, } \\
& d \text { is the distance between the dislocation } \\
& \text { and the surface. }
\end{aligned}
$$

The local stress can be approximately taken as equal to (3-2):

$$
\sigma=\frac{\mu b}{4 \pi R} \text {. }
$$

The image forces can be neglected when they are lower than the local stress. This condition $\sigma_{\mathrm{i}} \ll \sigma$ is fulfilled when $d \geq R\left(\sigma_{\mathrm{i}} \leq \sigma / 4 \pi\right)$. Consequently, local stress measurements must be performed only on dislocations at distances of more than $R$ from the surfaces.

4.1.2 Dislocation positions. - In a thin foil, under stress, dislocations take a position which depends on their line tension, the applied stress, the interaction with the crystal lattice and the image forces. Either to minimize their energy by reducing their length, or because of the effect of the image forces, it is therefore possible that dislocations take some artefact positions. This is expected to occur mainly when the lattice friction is small.

Dislocations parallel to the foil plane can be eliminated rapidly at the surfaces and the density of moving dislocations can be smaller in a thin foil than in the bulk for a given strain rate. 
4.1.3 Pinning effect. - The emergence points of moving dislocations are submitted to frictional forces originated from the formation of surface steps. Quantitative evaluations of this force are not available in literature. The analysis of dislocation shapes seems however to show that the curvature of dislocations resulting from this pinning force extends only over a very short length.

4.1.4 Kink, macrokink or cross-slip nucleation at the surface. - Kinks, macrokinks and cross-slip events can be nucleated at the free surface and some movements resulting from this artefact can occur. The corresponding driving force is a gain of energy by a reduction of the dislocation length.

4.1.5 Radiation effects. - Radiation damages resulting from the electron beam can lead to some artefacts. Radiation damages are obviously less important when the voltage is below the threshold voltage of the material and/or when the temperature is higher than a critical temperature linked to point defect mobility [29]. Following these considerations, feasibility diagrams for in situ deformation experiments have been established for a few cases [29]. At present, most experiments are performed in low voltage electron microscopes operating below the threshold voltage. Influence of point defect migration cannot however be eliminated unambiguously even under these favorable conditions.

4.2 HOW TO CHECK THE VALIDITY OF IN SITU DEFORMATION EXPERIMENTS. - It is clear that the artefacts mentioned above cannot be always ruled out unambiguously and each time an in situ deformation study is undertaken the problem of the validity of the results has to be addressed. From our experience in this field and from the knowledge of these artefacts a set of way to check this valiuity has been developed.

First, to eliminate the different surface effects in 4.1.1 to 4.1.4, it is always tried to check that dislocations which are not connected to free surfaces have the same behaviour as the others. These dislocations can either be part of a loop growing in the thin foil, or be anchored by obstacles at both extremities. Unambiguous examples can be found in [23] (Fig. 5), [28] (Fig. 4c and 5), [31] (Fig. 1 and 2), [32] (Fig. 3), [33] (Fig. III.11) and [34] (Fig. 11).

Second, the results obtained in situ are always compared with those obtained using other techniques, mainly macroscopic mechanical tests and post-mortem observations. As mentioned previously, the similarity between the temperature variation of the local stress and the macroscopic CRSS (see for instance Fig. 5, [18-22]) is probably the best way to establish the validity of in situ results. In the case of the prismatic glide of magnesium [30,31], systematic consistency has been found between the microscopic measurements of the local stress, activation area and activation enthalpy on one hand and the corresponding macroscopic values on the other. An excellent correlation in the superdislocation structure and behaviour in $\mathrm{L1}_{2}$ ordered alloys has been found between the results obtained by in situ deformation experiments and post-mortem T.E.M. observations. In the case of the cube glide of $\mathrm{L}_{2}$ alloys, dislocations in the same double Lomer and super Lomer configurations have been observed in situ in [32] and post-mortem in [35] using the weak-beam and lattice imaging technique. In the case of the octahedral glide of $\mathrm{L}_{2}$ alloys, the in situ observation of APB jumps ([33, 34] and Fig. 4 of [36] this issue) has been confirmed by a statistical analysis of the post-mortem weak-beam pictures [37].

\section{Information obtained by in situ deformation experiments.}

5.1 DETERMINATION OF THE DEFORMATION MODES. - The orientation of the observed specimen area is determined by conventional diffraction methods and represented with the help of a stereographic projection. Defects which accommodate the deformation are identified and characterized using usual methods developped for T.E.M.. For instance in the case of perfect disloca- 
tions, the Burgers vector and the character (screw, edge, $60^{\circ}, \ldots$ ) can be determined. In the case of twinning, the Burgers vector of the partial dislocations, the nature of the stacking fault and the degree of perfection of the microtwin can be analysed [38-40]. As discussed in another paper [36], the use of the weak-beam technique during in situ deformation experiments allows us to analyse dislocation processes at a very small scale in correlation with the core structure.

5.2 TRACE ANALYSIS. - The analysis of traces left on the two specimen surfaces by the moving dislocations gives also very fruitfull information. Because the thin foil surface is not absolutely clean, the shearing due to the dislocation movement is partly impeded. This produces a defect (the trace) locked under the surface, which is equivalent to a dislocation with a Burgers vector equal to a fraction of that of the moving dislocation. If follows that this linear defect lies at the intersection between the displacement plane and the foil surfaces, and that the extinction rules of the traces are the same as those of the moving dislocations [41]. The analysis of these traces may lead to the determination of the nature of the movement. Glide movements of screw dislocations lead to two traces which related to each other by a translation parallel to the Burgers vector [42]. This last property is no longer true in case of climb [42]. In case of glide, the glide plane can be determined from the direction of the traces and the occurrence of cross-slip will be checked from the wavy, serrated or rectilinear aspect of the traces.An example of slip traces is shown in figure 6 in the case of the prismatic glide of magnesium [30-31]: the two traces left by the dislocation can be related to each other by a translation parallel to the Burgers vector and the occurrence of cross-slip is demonstrated by the wavy nature of the traces.

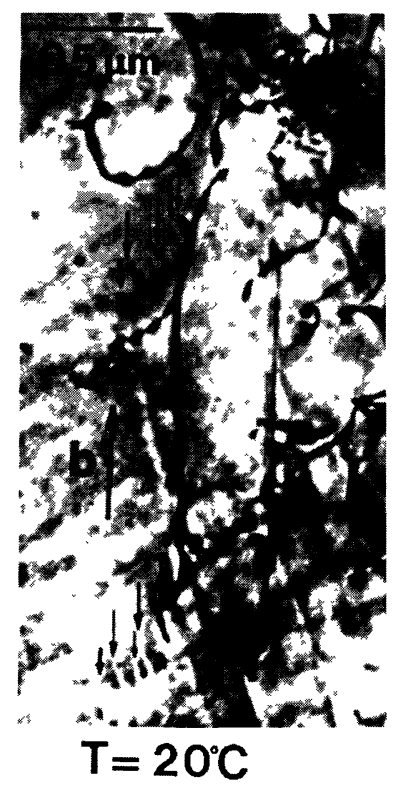

Fig. 6. - Example of slip traces in the case of the prismatic glide of magnesium. 
5.3 DeterminATION OF THE NATURE OF OBSTACLES. - The nature of the obstacles opposing to the movements of dislocations can also be determined.

First, these obstacles may be due to the lattice friction (intrinsic obstacles). All the mechanisms derived from the Peierls-Nabarro theory belong to this group of obstacles. In this case, the use of the in situ deformation technique has been found to be very fruitfull [9, 12, 18-24, 28, 30-34]. For more details, the reader is referred to two recent reviews [43,44]. An example is shown in figure 7 where a screw dislocation is moving by a serie of jumps between Peierls valleys in the case of the cube glide of the $\gamma^{\prime}$ phase of a superalloy at $570 \mathrm{~K}[12,25,28]$.

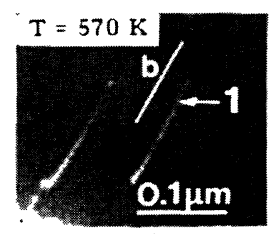

$\mathrm{t}=0$ to $0.40 \mathrm{~s}$.

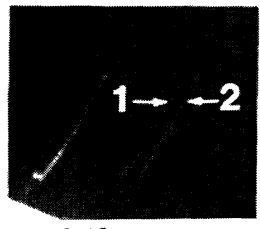

$\mathrm{t}=0.42 \mathrm{~s}$.

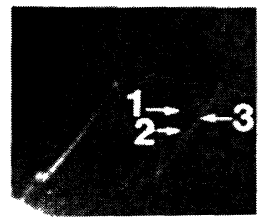

$t=0.4+4$.

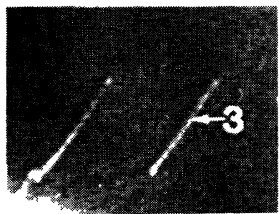

$\mathrm{t}-0.46 \mathrm{~s}$.

Fig. 7. - Sequence showing a screw dislocation moving by a serie of jumps between Peierls valleys in the case of the cube glide of the $\gamma^{\prime}$ phase of a superalloy at $570 \mathrm{~K}$.

Second, these obstacles may be extrinsic to the dislocations. By-passing of precipitates has been observed in [45]. Several examples of intersections between different deformation modes have been analysed: interactions between perfect dislocations and microtwins $[46,21,47]$ and twin-twin interactions [48]. In these cases, the reactions between the involved dislocations have been determined and their kinetics analysed. Figure 8 shows an example of a twin (A) which overcomes another twin (B) in a Cu-6.5 at. \%Si alloy at $480 \mathrm{~K}$ [48]. In this case, it has been shown that this overcoming results from the opening of a window in the twin obstacle by the first partial dislocations of the incident twin. Similar studies have been performed in the cases of interactions between a microtwin and a twin boundary [49] and between perfect dislocations and either a subgrain [36] or a grain boundary [50]. In the case of these extrinsic obstacles, much information has also been given by in situ deformation experiments.

5.4 DifFERENT ASPECTS OF THE MOVEMENT. - During an in situ test, the aspect of the active deformation processes can be qualitatively characterized in different ways.

The deformation may be accomodated by individual or collective movements of dislocations. For instance, dislocation may have an individual movement controlled by their own interaction with the lattice $[42,43]$. In the cases of subboundary migration during creep [36, 58-60] or of twinning [38-40], the deformation is controlled by the collective movement of groups of dislocations.

Dislocation movements may be steady or jerky. For instance, in the case of Peierls frictional forces, depending on the material and/or the temperature investigated, steady or jerky movements interpreted in terms of the Peierls mechanism or the locking-unlocking mechanism, respectively, have been observed [42, 43].

The deformation may also be homogeneous (movement of individual dislocations) or heterogeneous (movement of group of dislocations in pile-ups). In the case of concentrated solid solutions, the deformation is very often observed to be heterogeneous. It proceeds through the rapid multiplication and propagation of several hundred of dislocations on the same slip plane [51]. In the case of nickel-chromium alloys, the strain localisation and the corresponding heterogeneity is 

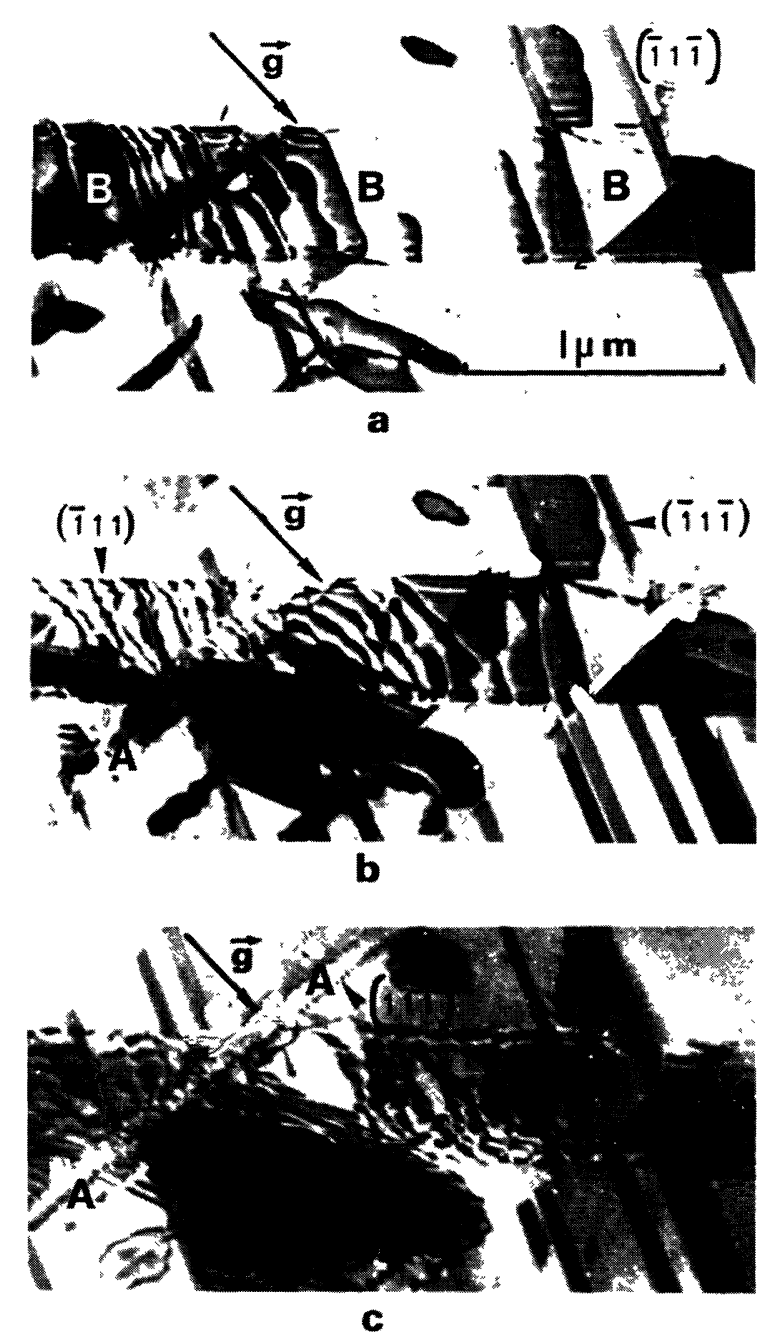

Fig. 8. - Sequence showing the overcoming of the twin B (111) by the twin A (111) in a Cu-6.5 at.\% Si alloy at $480 \mathrm{~K}$.

attribuated to a glide softening mechanism which is correlated to the progressive destruction of the short range order domains existing in these alloys [52].

In the case of extrinsic obstacles, the crossing may be easy, difficult or impossible. For instance, in the case of the simple dislocation-twin interaction in $\mathrm{TiAl}$, simple dislocations cannot cross twins whereas Shockley dislocations forming the twin can carry on to move after some interaction processes with simple dislocations [21, 47].

5.5 QUANTITATIVE MEASUREMENTS. - Depending on the physical problem under investigation, it is possible to measure some quantities in order to characterize the deformation. Most of them are relevant to the kinetics of the dislocation movements. 

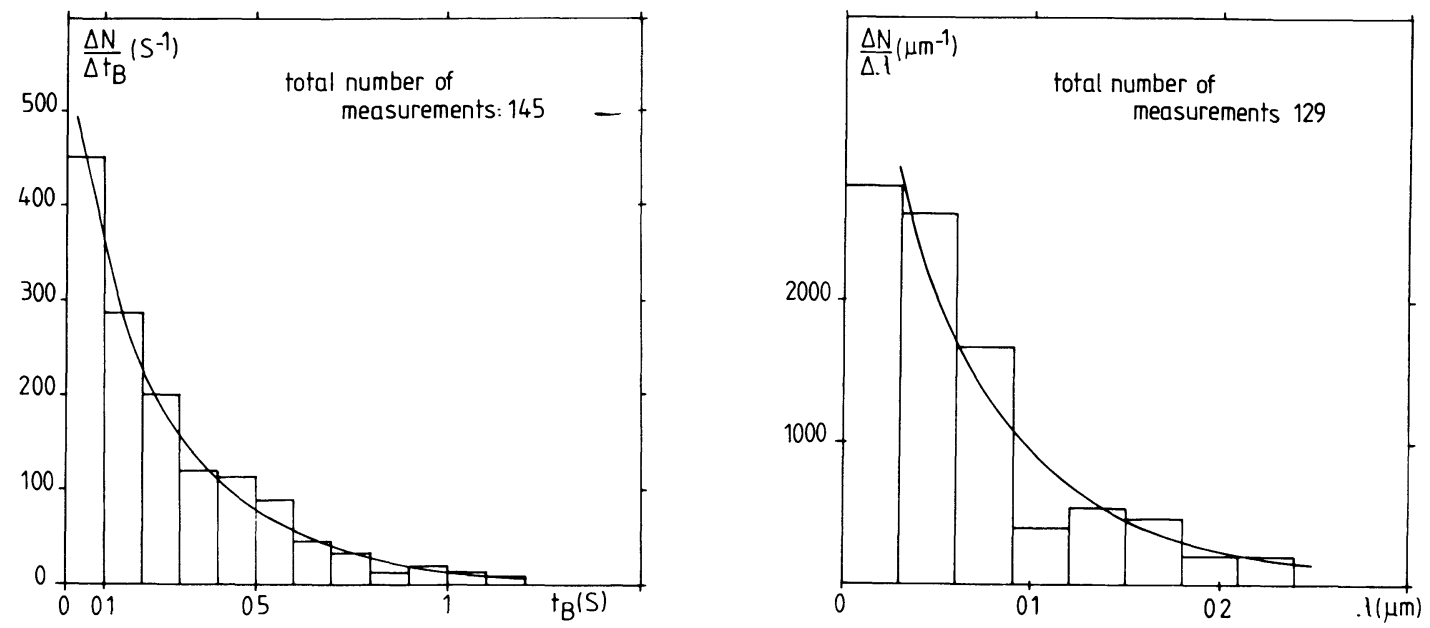

Fig. 9. - Histograms of the waiting times and of the jump lengths for the case of the prismatic glide of beryllium.

First, the velocity of moving dislocations can be measured. Depending on the obstacle distribution the velocity will be more or less heterogeneous. For instance, in the case of the prismatic glide of magnesium $(T \geq 300 \mathrm{~K},[30,31])$ in which the deformation is controlled by the Peierls mechanism, dislocations have to overcome obstacles resulting from their core spreading out of their glide plane on each atomic row. At the scale of our observations, the obstacle distribution appears to be regularly spaced and the velocity is constant over a given dislocation length. Measurements of the average velocity have been performed with a good accuracy in this case. When the distribution of obstacles is heterogeneous and/or the corresponding waiting time for crossing is not constant, only an average velocity over long distances with respect to the obstacle separation distance can be measured. The distribution of these measurements is obviously wider than in the previous case. In the latter case, rather than measuring the velocity of dislocations, it is often more fruitfull to measure the obstacle distribution and the corresponding waiting time. This has been done in some materials in which the deformation is controlled by the locking-unlocking mechanism [18-20, 12, 25, 28]. Obstacles result in this case from a thermally activated cross-slip of dislocations into a stable, sessile configuration and therefore their distribution, which is controlled by the cross-slip probability, is not constant. The waiting time which is also described by a probability law is not constant for the same reason. The kinetics of this movement has been studied by performing a statistical analysis of the displacement of a large number of dislocations. That means that all the waiting time and all the jump lengths have been measured carefully. The histograms of the jump lengths and of the waiting times give the average distribution of the obstacles and the average overcoming time, respectively. From the evolution of these parameters a model for the yield stress anomaly has been set up. These histograms are presented in figure 9 for the case of the prismatic glide of beryllium [18-20].

In the case of the extrinsic obstacles mentioned in 5-4, in principle the same procedure can be used. Because this statistical method requires a large number of measurements, it is practically impossible to use it and it is only to make a comparison between the movement time between the obstacles and those of waiting time in order to reveal what is controlling the dislocation movement.

The dislocation length can also be measured. It is therefore possible to check the validity of the 
frictional force models by measuring the velocity of the dislocations proportional to their length (length effect) [53, 18-22, 54]. The density of moving dislocations and the strain rate can also be determined using this technique. Local strain rate has been found to be always about $10^{-6}$ $10^{-4} \mathrm{~s}^{-1}\left(10^{-7}\right.$ in creep) during successful in situ deformation experiments. Indeed, a higher strain rate will lead to dislocations moving too fast to be observed and measured, whereas a smaller one will lead to a too small number of moving dislocations.

As a conclusion, in this paragraph a few examples of quantitative measurements have been given. Others examples are the measurements of distances between neighbouring in pile-ups [22, 40] and the measurements of dislocation dissociations under load in $\mathrm{Ni}_{3} \mathrm{Al}[55,33,34]$ and $\mathrm{Ti}_{3} \mathrm{Al}$ [56].

5.6 ACTIVATION PARAMETERS. - When the temperature, the local stress and the kinetics of the movement are known, the activation parameters can be directly measured. The activation area can be obtained by measuring the local stress variation as a function of the variation of either the dislocation velocity, or the strain rate during a relaxation test. It is given by:

$$
\begin{aligned}
& A=\frac{k T}{b} \frac{\Delta \log V}{\Delta \sigma} \quad \text { where : } A \text { is the activation area, } \\
& k \text { is the Boltzman's constant, } \\
& b \text { is the Burgers vector, } \\
& T \text { is the temperature, } \\
& V \text { is the dislocation velocity, } \\
& \sigma \text { is the local stress. }
\end{aligned}
$$

The activation enthalpy $(\Delta H)$ can be obtained by doing similar measurements at different temperatures. It is given by:

$$
\Delta H=\frac{-\Delta \log V}{\Delta(1 / k T)}
$$

One example of such measurements is shown in figure 10 for the case of the prismatic glide in magnesium. Over large ranges of local stress and temperature, the strain rate and the activation parameters have been measured $[18,23,30,31,60]$. Similar measurements have been performed in semi-conductors [54]. The activation enthalpy of super-Shockley dislocation motion has been determined in the $\gamma^{\prime}$ phase of the CMSX2 superalloy between $675^{\circ} \mathrm{C}$ and $920^{\circ} \mathrm{C}$ by measuring the kinetics of growth of the SISF [57].

Such evaluations of the activation parameters cannot always be performed. In fact, they can be performed only when the stress dependance is relatively small (A between 10 to $20 \mathrm{~b}^{2}$ ) because a small variation of the dislocation velocity (or strain rate) which can be measured accurately corresponds to a large variation of the local stress which is more difficult to estimate. When such measurements are not possible, and estimate of the stress dependence of the deformation mode observed can be deduced from the amplitude and duration of the relaxation.

\section{Discussion.}

In this article in situ deformation experiments have been shown to give usefull information on the microscopic behaviour of defects in order to explain the macroscopic mechanical properties of materials. This requires performing experiments with good straining holders on very good 


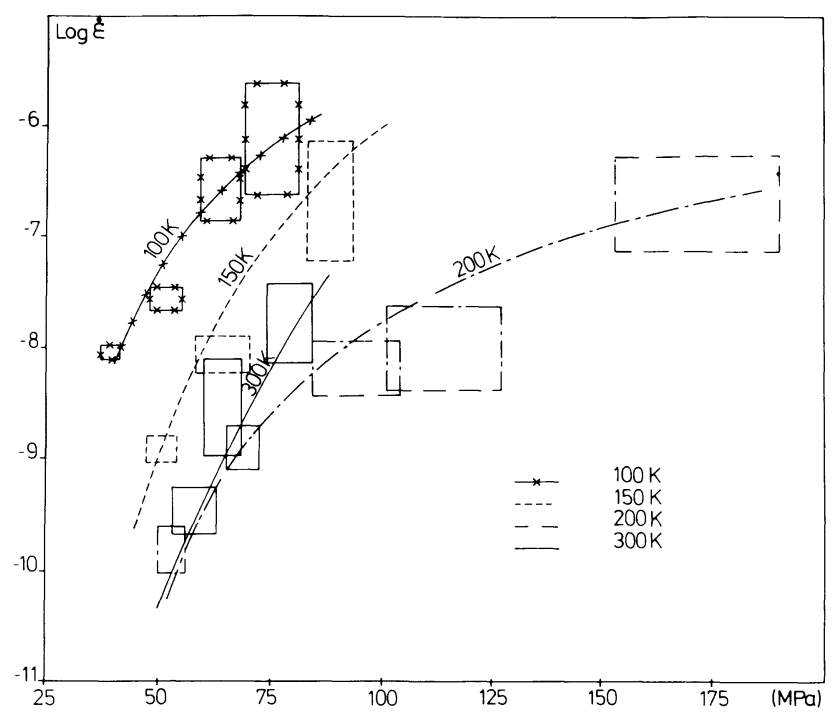

Fig. 10. - Variations at different temperatures of the local strain rate versus the local stress for the case of the prismatic glide of magnesium.

samples and to analyse the results carefully. Quantitative measurements should be done whenever possible.

In such conditions this technique appears to be essential since it is the unique giving direct information about dislocation behaviour under stress and at different temperatures. It is however obvious that it should be used in combination with other experimental techniques like macroscopic mechanical tests and post-mortem T.E.M. observations. It is however worth noting that the T.E.M. in situ tests often allows to correlate the results obtained by mechanical tests and by post-mortem analysis. Computer simulations of the defect core structure can also be performed to complete the investigation of the mechanical properties.

\section{Conclusion.}

In this paper the potentiality of the in situ deformation technique has been emphasized.

Some technical points which must be observed for the realisation of good experiments have been described: it is essential to build good performant straining holders and to prepare very good specimens.

Ways to control the stress, the temperature and the validity of the results have been described and it can be concluded that with a good knowledge of these problems fruitfull information can be obtained with this technique.

Many examples of results obtained by in situ tests have been reviewed briefly and the many capabilities of this technique have been demonstrated. A set of quantitative methods to characterize the defect movements which have been developed in Toulouse during the last few years has been presented.

The in situ deformation technique appears to be an essential tool in the field of the study of the mechanical properties which must be used in conjonction with other investigation methods. 


\section{References}

[1] Hirsch P.B., Horne J.W. and Whelan M.J., Phil. Mag. 1 (1956) 677.

[2] WILSDORF H.G.F., ASTM Spec. Tech. Publ. 245 (1958) 43.

[3] Fujita H., Electron Microsc., ed. R. Uyeda, (Maruzen, Tokyo, 1966) p. 289.

[4] Furubayashi E., J. Phys. Soc. Japan 27 (1969) 130.

[5] LOUCHET F. and Kubin L.P., Scripta Met. 9 (1975) 911.

[6] CAILlaRd D. and MARTIN J.L., Proceedings of Fourth International Congress on H.V.E.M., (Toulouse, France 1975) 305.

[7] VESELY D., Phys. Stat. Sol. 29 (1968) 675.

[8] Kubin L.P. and VeYsSiÈre P., Electron Microsc. 82, Proc. 10th Int. Cong. on Electr. Micros. (Hamburg, 2 1982) 531.

[9] Couret A. and CAillard D., Acta Met. 36 (1998) 2515.

[10] PÉLISSIER J. and LOUCHET F., same conference.

[11] Coujou A., Lours P., Roy N.A., Caillard D. and Clément N., Acta Met. 38 (1990) 825.

[12] Clément N., Molénat G. and Caillard D., Phil. Mag. 64 (1991) 697.

[13] HIRTH J.P. and LOTHE J., Theory of dislocation (Wiley-Interscience Publication, 1982).

[14] DouIN J., private communication (1991).

[15] Douin J., Veyssière P. and Beauchamp P., Phil. Mag. A 54 (1986) 375.

[16] Louchet F., Thèse d'Etat, Université Paul Sabatier (Toulouse-France, 1976).

[17] Howie A. and Swann P.R., Phil Mag. 6 (1961) 1215.

[18] Couret A., Thèse de Doctorat, Université Paul Sabatier (Toulouse, 1989).

[19] Couret A. and Caillard D., Phil. Mag. 59 (1989) 783.

[20] Couret A. and CAillard D., Phil. Mag. 59 (1989) 801.

[21] FARENC S., Thèse de Doctorat, Université Paul Sabatier (Toulouse-France, 1992).

[22] Farenc S., Caillard D. and Couret A., Accepted in Acta. Met. Mater (1993).

[23] Couret A., Caillard D., Puschl W. and Schoeck G., Phil. Mag A, 63 (1991) 1045.

[24] Fnaiech M., Reynaud F., Couret A.and Caillard D., Phil. Mag. $A 55$ (1987) 405.

[25] ClÉment N., Lours P., Coujou A. and CAIllard D., Proceedings of ICSMA 9, Haifa, Israel, Eds. D.G. Brandon, R. Chaim and A. Rosen, Freund Publishing House 1 (1991) 327.

[26] MARTIN J.L. and KUBIN L.P., Ultramicroscopy 3 (1978) 215.

[27] VEYSSIÈRE P., M.R.S. Symposium Proceedings on High Temperature Ordered Intermetallic Alloys 133 (1989) 175.

[28] Clément N., Couret A. and Caillard D., Phil. Mag. A 64 (1991) 669.

[29] Martin J.L. and Kubin L.P., Phys. Stat. Sol. 56 (1979) 487.

[30] Couret A. and CaIllard D., Acta Met. 33 (1985) 1447.

[31] Couret A. and CAIllard D., Acta Met. 33 (1985) 1455.

[32] Molénat G. and Caillard D., Phil. Mag. A, 65 (1992) 1327.

[33] MolénAT G., Thèse de Doctorat, Université Paul Sabatier (Toulouse 1991).

[34] Molénat G. and Caillard D., Phil. Mag. A. 64 (1992) 1291.

[35] Sun Y.Q., HAZZLEDine P.M., CRIMP M.A. and COURET A. 64 (1991) 311.

[36] Caillard D., Couret A., Molénat G. and Crestou J., same conference.

[37] Couret A., Sun Y.Q. and Hirsch P.B., Accepted in Phil. Mag. (1993).

[38] Coujou A., Acta. Met. 31 (1983) 1505.

[39] Coujou A., Bénéteau A. and Alcouffe G. Acta Metall. 36 (1988) 1537.

[40] Farenc S., Coujou A. and Couret A., Accepted in Phil. Mag. (1993).

[41] Howie A. and Whelan M.J., Proc. Roy. Soc., A262 (1962) 206.

[42] Caillard D. and Martin J.L., Proc. of 7th H.V.E.M., Berkeley, Ed. R.M. Fisher, R. Gonsky, K.H. Westmacott (1983) 205.

[43] Couret A. and Caillard D., Proc. Int. Conf. on Dislocation Mechanisms and the Strength of Advanced Materials, Aussois, France, J.Phys. III (1991) 885.

[44] Caillard D., Couret A., Clément N., Farenc S. and Molénat G., Proceedings of ICSMA 9, Haïfa, Israel, Eds. D.G. Bradon, R. Chaim and A. Rosen, Freund Publishing House, 1 (1991) 139.

[45] Grosbras M., Denanot M.F., Dedieu E., Cahoreau M., Caillard D. and Martin J.L., Proc. of the fourth I.C.S.M.A. conference, Nancy, France, Ed. Lab. Phys. Sol. Phys. Sol. ENSMIM (1976) 244.

[46] Coujou A., Bénéteau A. and Clément N., Scripta. Metall. 19 (1985) 891.

[47] Farenc S., Coujou A. and Couret A., Accepted in J. Mater. Sci. Eng. A. (1993). 
[48] Coujou A., Phil. Mag. Let. 56 (1987) 13.

[49] Coujou A., Bénéteau A. and Clément N., Acta Metall. Mater, 40 (1992) 337.

[50] Michaud H.M., Baillin X., PÉlissier J., PutauX J.L. and Thibault J., same conference.

[51] Clément N., Coujou A., Coulomb P. and Martin J.L., Rev. Phys. Appl. 15 (1980) 831.

[52] Clément N., Caillard D. and Martin J.L., Acta Metall. 32 (1984) 961.

[53] Louchet F., Kubin L.P. and Vesely D., Phil. Mag. A 39 (1979) 433.

[54] Louchet F., Pélissier J., Caillard D., Peyrade J.P., Levade C. and Vandershaeve G., same conference.

[55] Caillard D., Clément N., Couret A., Lours P. and Coujou A., Phil. Mag. Lett. 58 (1988) 263.

[56] Legros M., Couret A. and CAillard D., Accepted in the M.R.S. Symposium Proceedings on High Temperature Ordered Intermetallic Alloys (1993).

[57] Lours P., Coujou A. and de MANDuIT B., Phil. Mag., 62 (1990) 253.

[58] Caillard D. and MarTin J.L., Acta. Met. 30 (1982) 791.

[59] Caillard D. and Martin J.L., Acta. Met. 31 (1983) 813.

[60] Caillard D., Phil. Mag. A 51 (1985) 157. 